\title{
Neutrinoless double beta decay search in XENON1T and XENONnT
}

\section{Maxime Pierre ${ }^{a, *}$ on behalf of the XENON collaboration}

${ }^{a}$ SUBATECH, IMT Atlantique, CNRS/IN2P3, Universite de Nantes, 4 rue Alfred Kastler, 44307 Nantes, France

E-mail: maxime.pierre@subatech.in2p3.fr

With the lowest background level ever reached by detectors searching for rare-events, XENON1T proved to be the most sensitive dark matter direct detection experiment on earth. The unprecedented low level of radioactivity reached, made the XENON1T experiment suitable also for other interesting rare-events searches including the neutrinoless double beta decay of ${ }^{136} \mathrm{Xe}$. In this proceeding I will report on the current status of neutrinoless double beta decay of ${ }^{136} \mathrm{Xe}$ search in XENON1T. Furthermore, in the context of the advancement of the XENON program, the next generation experiment, XENONnT, designed with a high level of background reduction aiming to increase the predecessor sensitivity in rare-events searches is currently under commissioning phase in the underground National Laboratory of Gran Sasso (LNGS): it hosts 5.9 tonnes of liquid xenon as a target mass. I will also discuss the ongoing sensitivity projection studies for neutrinoless double beta decay search in XENONnT.

$37^{\text {th }}$ International Cosmic Ray Conference (ICRC 2021)

July 12th - 23rd, 2021

Online - Berlin, Germany

\footnotetext{
${ }^{*}$ Presenter
} 


\section{Introduction}

The experiments conceveid and built by the XENON Collaboration are primarily devoted to the search for Weakly Interacting Massive Particles (WIMPs) Dark Matter (DM) candidates through the observation of the possible scattering of a WIMP off xenon nuclei. XENON1T hold the best limit for WIMP-nucleus cross-section [1]. With the improvement of the background reduction and the increase of the target mass from XENON10 to XENONnT, these experiments start to be sensitive to other rare events physics channels: among others, this has been proven in XENON1T by the observation of two-neutrino double electron capture on ${ }^{124} \mathrm{Xe}$ [2], the search for coherent elastic scattering of solar ${ }^{8} \mathrm{~B}$ neutrinos [3] and electronic recoils at low energies [4]. Furthermore, another particularly interesting search carried on by the XENON Collaboration is represented by the neutrinoless double beta decay $(0 v \beta \beta)$ of ${ }^{136} \mathrm{Xe}$.

While the two-neutrino double beta decay $(2 v \beta \beta)$ is a rare nuclear transition allowed in the Standard Model (SM), the $0 v \beta \beta$ would lead to the violation of the lepton number and it is thus forbidden in the SM. Such observation would shed light on the nature of neutrinos by indicating that they are Majorana fermions, i.e. their own anti-particles. The $2 v \beta \beta$ was first suggested by M. Goeppert-Mayer in 1935 [7]: it consists in a nucleus emitting two electrons and two antineutrinos $\left(\overline{v_{e}}\right)$, as shown in the left of Fig. 1. The $2 v \beta \beta$ decay has been already observed in several isotopes. The $0 v \beta \beta$ decay has been theorised by W. Fury in 1939 [8]. The basic mechanism induced by the exchange of a light Majorana neutrino is shown in Fig. 1 on the right. One of the most studied isotope in the quest for the $0 v \beta \beta$ decay is the ${ }^{136} \mathrm{Xe}$, which is present in the target of the XENON detectors with a natural abundance of $8.9 \%$. This isotope has already shown to undergo $2 v \beta \beta$ with a half-life of $\left(2.165 \pm 0.016^{(\text {stat })} \pm 0.059^{(\text {sys })}\right) \times 10^{21}$ years with a $Q_{\text {value }}$ of $(2457.83 \pm 0.37)$ $\operatorname{keV}[9,10]$ and the current best limit on the half-life of the $0 v \beta \beta$ decay of ${ }^{136} \mathrm{Xe}$ is hold by the KamLAND-Zen experiment with a $T_{1 / 2}^{0 v}>1.07 \times 10^{26}$ years [11].

In this proceeding I will firstly present the design of the XENON1T and XENONnT experiments and their working principle in section 2. The signal and backgrounds models considered for $0 v \beta \beta$ decay search will be introduced in section 3 and the development of the $0 v \beta \beta$ decay data analysis for XENON1T and the sensitivity projection study for XENONnT will be covered in section 4. I will finally summarize the work discussed in this contribution in section 5 .

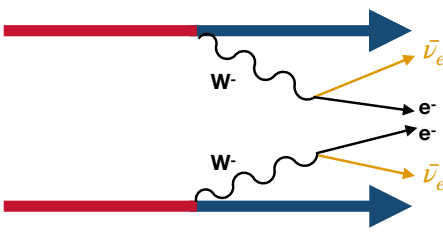

$(A, Z) \longrightarrow(A, Z+2)+2 e^{-}+2 \bar{\nu}_{e}$

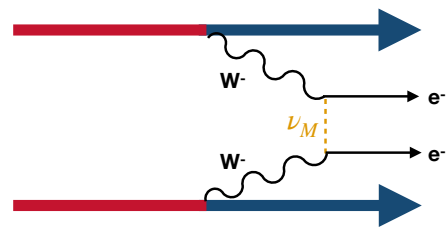

$(A, Z) \longrightarrow(A, Z+2)+2 e^{-}$

Figure 1: Feynman diagrams of $2 v \beta \beta$ decay (left) and $0 v \beta \beta$ decay for light Majorana neutrino exchange mechanism (right). 


\section{The XENON Collaboration experiments}

The XENON dark matter program started in 2005 with the XENON10 detector followed by XENON100 and XENON1T [13-15]. Those experiments were operating at the Istituto Nazionale di Fisica Nucleare (INFN) Laboratori Nazionali del Gran Sasso (LNGS) in Italy, an underground laboratory with a 3600 m.w.e depth that reduces the surface muon flux by a factor of $10^{6}$. At present, the forth generation experiment, XENONnT, is under commissioning phase at the same laboratory. All the above mentioned experiments are based on the same detection technology consisting of a dual-phase Time Projection Chamber (TPC) filled with Liquid (LXe) and Gaseous Xenon (GXe).

The working principle of such detector is the following: when a particle interacts with the detector target, it can either scatter off LXe nuclei (in case of WIMPs, neutrons or neutrinos) or atomic electrons ( $\gamma$ rays, charged particles and electronic neutrinos), generating Nuclear (NR) or Electronic Recoils (ER), respectively. The recoil will excite and ionize encountered LXe atoms, causing the release of photons and electrons as it is illustrated in Fig. 2 on the left. Photons will firstly be detected via Photomultipliers tubes (PMTs) on the top and the bottom of the TPC: this first scintillation signal is called S1. Ionized electrons will follow the electric field within the TPC, drifting towards the liquid-gas interface. This will lead to the emission of a second scintillation signal, called S2, in the gaseous phase. Events can be reconstructed in three dimensions using the drift time between $\mathrm{S} 1$ and $\mathrm{S} 2$ to reconstruct the $\mathrm{z}$ coordinate and the $\mathrm{S} 2$ hit pattern on the top PMT array for the $(\mathrm{x}, \mathrm{y})$ coordinates. The S1/S2 ratio can be used to distinguish between the two different types of recoils which is primordial to distinguish background events from the expected WIMP signals. Thanks to its high stopping power for gamma and beta radiations (resulting in a self-shielding from external backgrounds) and to the low contamination of long-lived radioactive isotopes (which minimize internal backgrounds), LXe represents an ideal target for WIMP searches.
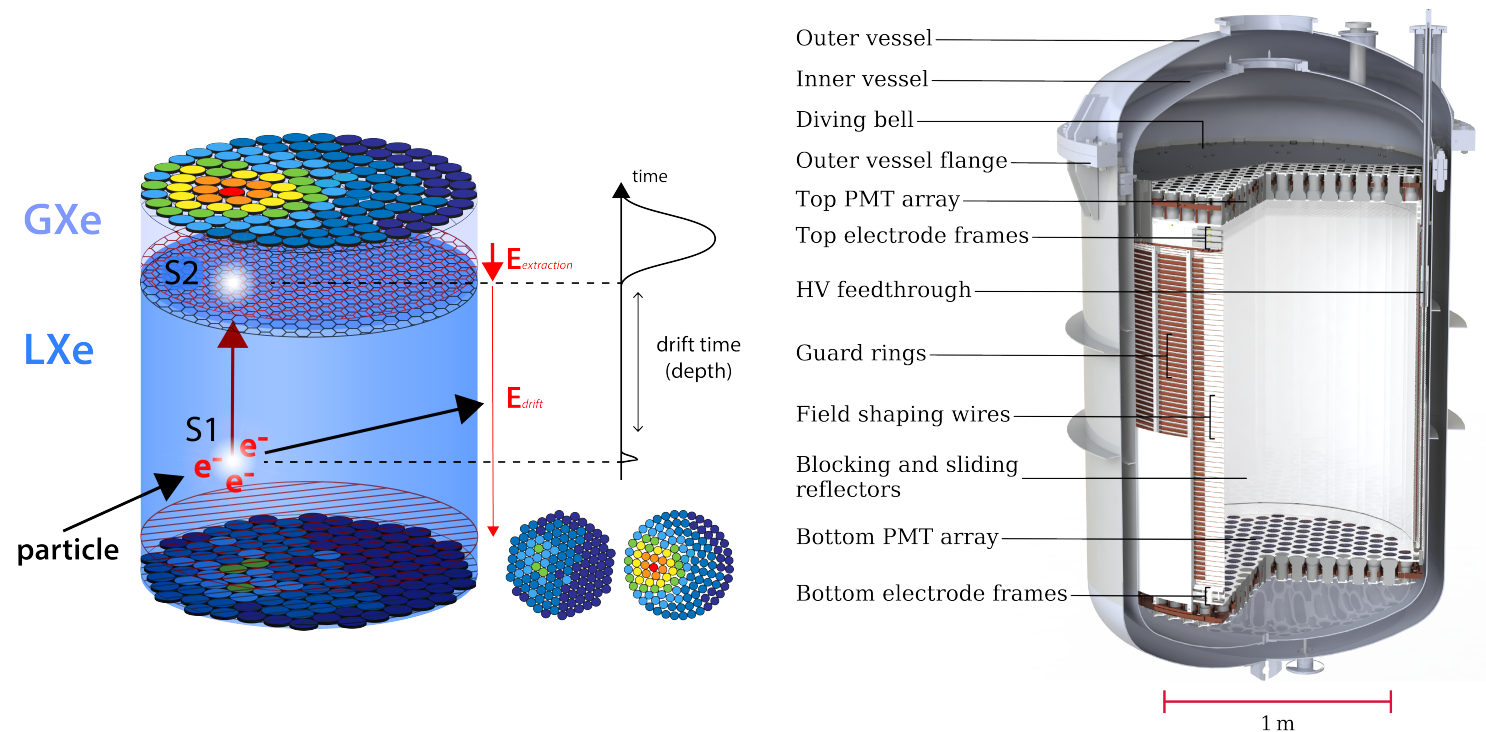

Figure 2: (Left) Working principle of a dual-phase TPC. (Right) CAD rendering of the XENONnT cryostat and TPC. 


\subsection{XENON1T}

XENON1T is the third generation of experiments of the XENON collaboration operating from 2016 to 2018. Being conceived for rare events searches, a careful selection of radiopure materials has been performed [16] in order to minimize the impact of radiogenic backgrounds. The heart of XENON1T is the TPC which measures $97 \mathrm{~cm}$ in high and $96 \mathrm{~cm}$ in diameter, designed to contain 2 tonnes of LXe used as a target in the instrumented active volume. Its boundaries are delimited by 24 polytetrafluoroethylene (PTFE) reflectors panels at the side and by gate and cathode electrodes at the top and bottom, respectively, that generate the drift field inside the detector. An anode electrode is placed in the GXe phase which generates a stronger electric field to accelerate and extract electrons from the liquid phase and produce S2 signals. The liquid-gas interface in the TPC is controlled by a Stainless Steel (StS) diving bell and it is placed slighlty above the gate electode. Two arrays of PMTs, one placed at the top and the other at the bottom of the TPC, are instrumented with 127 and 121 Hamamatsu R11410-21 3" PMTs respectively, chosen for their low radioactivity and high quantum efficiency $(\mathrm{QE} \approx 34 \%$ on average at room temperature) at the xenon scintillation wavelength of $175 \mathrm{~nm}$ [17]. The TPC is encapsulated in a double-walled vacuum-isolated StS cryostat surrounded by an active water Cherenkov muon veto system [18] also used as a passive shield for external backgrounds sources. The xenon is continuoulsy cooled and purified thanks to cryogenic and GXe purification systems located outside of the water tank and connected to the cryostat by a large vacuum-insulated cryogenic pipe. Furthermore, a dedicated xenon-storage system called ReStoX (Recovery and Storage of Xenon), has been conceived and realize to store up to 7.6 tonnes of xenon either in liquid or in gas phase, to fill the xenon into the detector vessel in ultra high purity conditions and to ensure a fast recovery of xenon in case of emergency.

\subsection{XENONnT}

While conceiving the XENON1T experiments, several subsystems such as the water tank, the muon veto, the outer cryostat vessel and the GXe purification system were designed to also host its successor XENONnT. This strategy allowed a quick transition between the two generations experiments. With the design of the XENONnT TPC, shown in Fig. 2 on the right, the size of the detector itself has increased up to $148 \mathrm{~cm}$ in height and $137 \mathrm{~cm}$ in diameter, with an active region of 5.9 tons of LXe. The purity level of the xenon target mass is improved compare to XENON1T thanks to a novel liquid purification system allowing for a continuous circulation of the LXe and a radon distillation column to further suppress radon backgrounds. A new neutron veto has been instaled and will enable the identification and rejection of otherwise irreducible neutron backgrounds, for WIMP searches, in the target volume. Given the larger xenon inventory a second storage system of LXe, ReStoX 2, has been installed. The expected sensitivity to spin-independent WIMP-nucleon interactions for XENONnT with an exposure goal of 20 ty is $1.4 \times 10^{48} \mathrm{~cm}^{2}$ for a $50 \mathrm{GeV} / \mathrm{c}^{2}$ mass WIMP at $90 \%$ confidence level [19].

\section{Signal and Background models}

The expected signature of the $0 v \beta \beta$ decay in our detector is a Single Scatter (or Single Site, SS) event whose energy corresponds to the sum of the two emitted electrons at the $Q_{v a l u e}$ of the 
process, noted $Q_{\beta \beta}$. Due to the high stopping power of LXe, the electrons penetration length in LXe is smaller than $3 \mathrm{~mm}$ : this explain why a SS event is expected. Multiple Scatter (or Multi Site, MS) events might instead occur when $\beta$-electrons or Bremsstrahlung photons generate multiple energy depositions. Monte-Carlo (MC) simulations have shown that the energy of the expected signal within an optimized Fiducial Volume (FV) and after having accounted for energy resolution effects and removed MS events, follows a gaussian distribution centered at the $Q_{\beta \beta}$.

Two different type of background sources can mimic the $0 v \beta \beta$ decay signature in our detector: the external and the internal ones.

\subsection{External Backgrounds}

The detector materials might contain long-lived radionuclides that might emanate into the target and introduce background. Those materials were carefuly chosen, based on their radiopurity, in order to minimize as much as possible the induced background for the WIMP search. A dedicated screening campaign has been carried on [16]. However, the natural decay chains of ${ }^{238} \mathrm{U},{ }^{235} \mathrm{U}$ and ${ }^{232} \mathrm{Th}$ might introduce ER background in the $0 v \beta \beta$ energy Region Of Interest (ROI) in the form of $\gamma$-rays that can produce low energy Compton scatters. In particular the ${ }^{214} \mathrm{Bi}$ and ${ }^{208} \mathrm{Tl} \gamma$ lines $\left({ }^{238} \mathrm{U}\right.$ and ${ }^{232} \mathrm{Th}$ daugthers) at $2447.9 \mathrm{keV}$ and $2614.5 \mathrm{keV}$ respectively, are close to the $Q_{\beta \beta}$ and their interaction in LXe can mimic the signature of a $0 v \beta \beta$ decay. Another source of background is represented by the ${ }^{60} \mathrm{Co}$ in two gammas with energies of $1173.2 \mathrm{keV}$ and $1332.5 \mathrm{keV}$ respectively. Being not temporarily resolvable, the two peaks could pileup and be reconstruced as a unique peak at $2505.7 \mathrm{keV}$. Nevertheless this background has been proven to be negligeable.

\subsection{Internal Backgrounds}

Internal backgrounds are either intrinsic to the liquid gas target or originate from interactions of cosmogenic particles with the xenon target. A list of internal background that are relevant for the $0 v \beta \beta$ search is provided below.

- $2 v \beta \beta$ of ${ }^{136} \mathrm{Xe}$ : Its continuous spectrum with the endpoint at the $Q_{\beta \beta}$ is a background for $0 v \beta \beta$ decay search. Theoretical calculations from J. Kotila and F. Iachello [12] were used in order to model this background. Thanks to the sub-percent energy resolution at $Q_{\beta \beta}$ demonstrated in XENON1T [5], its contribution in the $\pm 1 \sigma$ ROI is negligible with respect to the material backgrounds for both XENON1T and XENONnT.

- ${ }^{222} \mathrm{Rn}$ contamination in LXe: Radon emanation from the materials of the detector to our LXe target induces a second contribution from ${ }^{238} \mathrm{U}$ primoridal decay chain products, in addition to the one from materials. Due to the particular topology of events from ${ }^{214} \mathrm{Bi} \beta$-decays into ${ }^{214} \mathrm{Po}$ quickly followed by ${ }^{214} \mathrm{Po}$ decays through an $\alpha$ emission into ${ }^{210} \mathrm{~Pb}$, when the process occurs in the instrumented detector, we can identify and thus reject it. This technique, so called BiPo tagging, can reach a rejection power $>99.8 \%$ [6]. However, when the decays occurs in the non-instrumented LXe outside of the active volume, the BiPo tagging cannot be used, and therefore the $2.45 \mathrm{MeV} \gamma$ from ${ }^{214} \mathrm{Bi}$ decay reaching the $\mathrm{FV}$ cannot be rejected and will represent a source of background. 
- $\beta$-decay of ${ }^{137} \mathrm{Xe}$ : With a $Q_{\text {value }}=4.17 \mathrm{MeV}$ far beyond the $Q_{\beta \beta}$, it is a relevant source of ER background for $0 v \beta \beta$ decay search. ${ }^{137} \mathrm{Xe}$ is produced through neutron capture on ${ }^{136} \mathrm{Xe}$ occuring either within the TPC itself or in the non-shielded parts (placed outside of the water tank) of the purification systems. The muon-induced neutrons produced in the LXe are the principle responsibles for the production of ${ }^{137} \mathrm{Xe}$ in the TPC, while the thermal neutrons flux induced by radiogenic decay from the rocks, concrete and materials has the stronger impact on the production of ${ }^{137} \mathrm{Xe}$ in the purification system.

- ${ }^{8} \mathrm{~B}$ solar neutrinos: $v$-electron scattering is an irreducible source of ER background in our detector that can be problematic for $0 v \beta \beta$ decay search if the incident neutrino flux and energy is sufficiently high. While the contribution from atmospheric neutrinos, diffuse supernovae neutrinos, or geo-neutrinos can be excluded since either their flux or their energy is too small, the contribution from ${ }^{8} \mathrm{~B}$ solar neutrinos can be relevant for our study.

\section{4. $0 v \beta \beta$ decay search in XENON1T and XENONnT}

Xenon dual-phase TPCs designed to search for WIMP DM candidates are optimised to work in the few $\mathrm{keV}$ electron recoil energy range. At energies above $\sim 100 \mathrm{keV}$, the signals measured by the PMTs start to saturate and the energy reconstruction is impacted. To allow the XENON experiments to simultaneously search for DM and $0 v \beta \beta$ decay of ${ }^{136} \mathrm{Xe}$, several improvements especially concerning the reconstruction and correction of high energy signals have to be made in the $\mathrm{MeV}$ region. In XENON1T these improvements led to the world leading energy resolution at $Q_{\beta \beta}$ of $0.8 \%(\sigma / \mathrm{E})$ in a xenon dual phase TPC [5], shown in Fig. 3. Based on this promising result, a blinded analysis to search for $0 v \beta \beta$ events is currently being performed using XENON1T data. As discussed in section 3, the expected $0 v \beta \beta$ signal in our detector is a ER event reconstructed as a SS. Selection criteria have been developed in order to maximize the signal acceptance while rejecting potential background interactions. These criteria are based on data quality checks, signals properties and reconstructions. Among them, the optimization of the FV (that depends on the target mass and the background rate in the volume) is a key element to increase the experimental sensitivity. The final post-unblinding results of the $0 v \beta \beta$ decay analysis in XENON1T will soon be published. A sensitivity projection study for $0 v \beta \beta$ decay search in XENONnT is ongoing. Thanks to a larger LXe active mass (x3) and a significant foreseen background reduction (x1/10), the sensitivity of XENONnT for $0 v \beta \beta$ decay search is expected to significantly increase with respect to its predecessor. Results of this sensitivity projection study for XENONnT will also be published soon.

\section{Summary}

XENON1T has proven to be sensitive to rare events searches on a wide range of energy from few kev to MeV. Despite not being competitive with currently leading double beta decay dedicated experiments, the results presented in this proceeding open the path towards a simultaneous search for DM and $0 v \beta \beta$ decay with current and next generation LXe dual phase TPCs such as XENONnT. 


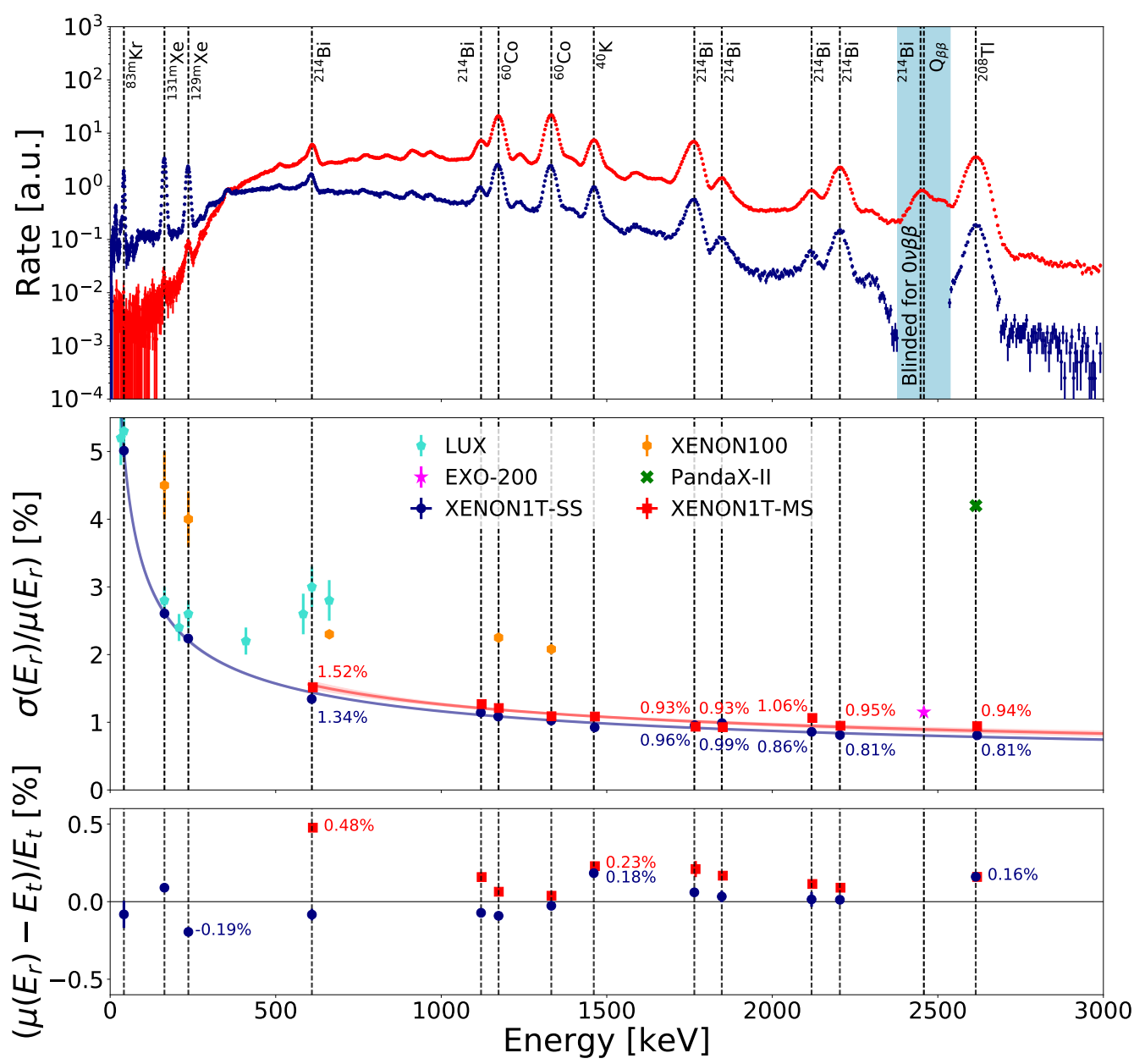

Figure 3: (Top) Electronic recoil energy spectra of Single Site (blue) and Multi Site (red) events in XENON1T for a $1 \mathrm{t}$ fiducial volume. SS events with energies around $Q_{\beta \beta}$ are blinded for the search for $0 v \beta \beta$ decay. The MS spectrum has a lower rate at low energies due to the fiducial volume selection. (Middle) The measured energy resolution for SS and MS events. The SS and MS resolutions as a function of energy are fit with $a / \sqrt{E}+b$ and shown by the blue and red lines, respectively, while the shaded regions cover $1-\sigma$ statistical uncertainty of the fits. (Bottom) The relative energy shift from the true values for SS and MS events.

\section{References}

[1] E. Aprile et al. (XENON Collaboration), Dark Matter Search Results from a One Ton-Year Exposure of XENONIT, Phys. Rev. Lett. 121 111302, (2018) [1805.12562].

[2] E. Aprile et al. (XENON Collaboration), Observation of two-neutrino double electron capture in ${ }^{124}$ Xe with XENON1T, Nature 568 532, (2019) [1904 . 11002].

[3] E. Aprile et al. (XENON Collaboration), Search for Coherent Elastic Scattering of Solar ${ }^{8} B$ Neutrinos in the XENON1T Dark Matter Experiment, Phys. Rev. Lett. 126 091301, (2021) [2012.02846]. 
[4] E. Aprile et al. (XENON Collaboration), Excess electronic recoil events in XENONIT, Phys. Rev. D 102 072004, (2020) [2006.09721].

[5] E. Aprile et al. (XENON Collaboration), Energy resolution and linearity of XENONIT in the MeV energy range, Eur. Phys. J. C 80 785, (2020) [2003.03825].

[6] L. Baudis et al., Neutrino physics with multi-ton scale liquid xenon detectors, JCAP 01044, (2014) [1309.7024].

[7] M. Goeppert-Mayer, Double Beta-Disintegration, Phys. Rev. 48 512, (1935).

[8] W. H. Furry, On Transition Probabilities in Double Beta-Disintegration, Phys. Rev. 56 1184, (1939).

[9] J. B. Albert et al. (EXO Collaboration), An improved measurement of the $2 v \beta \beta$ half-life of ${ }^{136}$ Xe with EXO-200, Phys. Rev. C 89 015502, (2014) [1306.6106].

[10] M. Redshaw et al., Mass and Double-Beta-Decay Q-Value of ${ }^{136}$ Xe, Phys. Rev. Lett. 98053003 , (2007).

[11] A. Gando et al. (KamLAND-Zen Collaboration), Search for Majorana Neutrinos Near the Inverted Mass Hierarchy Region with KamLAND-Zen, Phys. Rev. Lett. 117 082503, (2014) [1605.02889].

[12] J. Kotila and F. Iachello, Phase space factors for double- $\beta$ decay, Phys. Rev. C 85034316 , (2012) [1209.5722].

[13] E. Aprile et al. (XENON Collaboration), Design and Performance of the XENON10 Dark Matter Experiment, Astropart. Phys. 34 679, (2011) [1001. 2834].

[14] E. Aprile et al. (XENON Collaboration), The XENON100 dark matter experiment, Astropart. Phys. 35 573, (2012) [1107.2155].

[15] E. Aprile et al. (XENON Collaboration), The XENON1T Dark Matter Experiment, Eur. Phys. J. C 77 881, (2017) [1708.07051].

[16] E. Aprile et al. (XENON Collaboration), Material radioassay and selection for the XENONIT dark matter experiment, Eur. Phys. J. C 77 890, (2017) [1705. 01828].

[17] E. Aprile et al. (XENON Collaboration), Lowering the radioactivity of the photomultiplier tubes for the XENON1T dark matter experiment, Eur. Phys. J. C 75 546, (2015) [1503. 07698].

[18] E. Aprile et al. (XENON Collaboration), Conceptual design and simulation of a water Cherenkov muon veto for the XENONIT experiment, JINST 9 P11006, (2014) [1406 . 2374].

[19] E. Aprile et al. (XENON Collaboration), Projected WIMP sensitivity of the XENONnT dark matter experiment, JCAP 11 031, (2020) [2007.08796]. 